\title{
Acoustic waves in ducts with sinusoidally perturbed walls and mean flow
}

\author{
Ali H. Nayfeh \\ Department of Engineering Science and Mechanics, Virginia Polytechnic Institute and State University, Blacksburg, \\ Virginia 24061 \\ (Received 29 November 1974; revised 10 January 1975) \\ An analysis is presented of the propagation of acoustic waves in a hard-walled duct with sinusoidally \\ perturbed walls and carrying mean flow. The results show that resonance occurs whenever the \\ wavenumber of the wall undulations is approximately equal to the difference between the \\ wavenumbers of any two propagating modes. It is shown that neither of the resonating modes could \\ exist in the duct without strongly exciting the other resonating mode.
}

Subject Classification: $20.45,20.60 ; 28.60$.

\section{INTRODUCTION}

We consider sound propagation in a two-dimensional hard-walled duct having a nonuniform cross section and carrying a uniform average mean flow. We assume the walls to have weak sinusoidal undulations. We assume the motion to be inviscid and irrotational so that it can be described by a potential function. We make lengths, velocities, and time dimensionless by using the average width of the duct $d$, the undisturbed speed of sound $c$, and the characteristic time $d / c$ as reference quantities. In dimensionless quantities, the walls of the duct are located by

$$
\begin{aligned}
& y=\epsilon \sin \left(k_{w} x\right), \text { lower wall, } \\
& y=1+\epsilon \alpha \sin \left(k_{w} x+\theta\right), \text { upper wall, }
\end{aligned}
$$

where $\epsilon$ is a small dimensionless parameter characterizing the weakness of the undulations, $k_{w}$ is the wavenumber of the undulations. The phase difference between the undulations of the two walls is $\theta$ and $\alpha$ is the amplitude ratio of the upper and lower wall undulations.

The dimensionless potential function $\phi(x, y, t)$ describing the total motion is governed by ${ }^{1}$

$$
\begin{aligned}
\nabla^{2} \phi-\frac{\partial^{2} \phi}{\partial t^{2}}= & \frac{\partial}{\partial t}(\nabla \phi)^{2}-(1-\gamma)\left[\frac{\partial \phi}{\partial t}+\frac{1}{2}(\nabla \phi)^{2}-\frac{1}{2} M^{2}\right] \nabla^{2} \phi \\
& +\frac{1}{2}(\nabla \phi \cdot \nabla)(\nabla \phi)^{2}
\end{aligned}
$$

where $\gamma$ is the specific heat ratio of the gas and $M$ is the Mach number of the average mean flow. For an inviscid fluid and hard walls, the flow is tangential to the wall; that is,

$$
\begin{aligned}
& \frac{\partial \phi}{\partial y}=\epsilon k_{w} \frac{\partial \phi}{\partial x} \cos \left(k_{w} x\right), \text { at } y=\epsilon \sin \left(k_{w} x\right), \\
& \frac{\partial \phi}{\partial y}=\epsilon \alpha k_{w} \frac{\partial \phi}{\partial x} \cos \left(k_{w} x+\theta\right), \text { at } y=1+\epsilon \alpha \sin \left(k_{w} x+\theta\right) .
\end{aligned}
$$

For the case of no-mean flow and linear sinusoidal motion, straightforward expansions of the form $\phi=\phi_{0}$ $+\epsilon \phi_{1}$ were obtained by Isakovitch ${ }^{2}$ when $\alpha=0$, by Samuels ${ }^{3}$ when $\alpha=1$ and $\theta=0$, and by Salant when $\alpha=1$. Unfortunately, all the above expansions are not uniformly valid because the correction term $\epsilon \phi_{1}$ dominates the zeroth-order term $\phi_{0}$ for frequencies near what Isako- vitch, Samuels, and Salant call the resonant frequencies. Nayfeh ${ }^{5}$ determined a uniform expansion for the case treated by Salant by using the method of multiple scales. ${ }^{6}$ In this paper, the latter analysis is extended to include the effects of a mean flow whose average is uniform, having a Mach number $M$.

\section{MEAN FLOW SOLUTION AND ACOUSTIC EQUATIONS}

We assume that $\phi$ is the sum of a steady mean part and an unsteady acoustic part so that it can be expanded as

$$
\phi(x, y, t)=\phi_{0}(x, y)+\delta \phi_{1}(x, y, t)+\cdots,
$$

where $\delta$ is a small dimensionless parameter characterizing the intensity of the sound. Substituting Eq. 6 into Eqs. 3-5 and equating the coefficients of $\delta^{\circ}$ and $\delta$ on both sides, we have:

$$
\begin{aligned}
& \text { Mean flow: Order } \delta^{0} \\
& \qquad \begin{array}{l}
\nabla^{2} \phi_{0}=-\frac{1}{2}(1-\gamma)\left[\left(\nabla \phi_{0}\right)^{2}-M^{2}\right] \nabla^{2} \phi_{0}+\frac{1}{2}\left(\nabla \phi_{0} \cdot \nabla\right)\left(\nabla \phi_{0}\right)^{2} \\
\frac{\partial \phi_{0}}{\partial y}=\epsilon k_{w} \frac{\partial \phi_{0}}{\partial x} \cos \left(k_{w} x\right), \text { at } y=\epsilon \sin \left(k_{w} x\right) \\
\frac{\partial \phi_{0}}{\partial y}=\epsilon \alpha k_{w} \frac{\partial \phi_{0}}{\partial x} \cos \left(k_{w} x+\theta\right) \\
\text { at } y=1+\epsilon \alpha \sin \left(k_{w} x+\theta\right)
\end{array}
\end{aligned}
$$

Acoustic field: Order $\delta$

$$
\begin{aligned}
\nabla^{2} \phi_{1}-\frac{\partial^{2} \phi_{1}}{\partial t^{2}}= & 2 \frac{\partial}{\partial t}\left(\nabla \phi_{0} \cdot \nabla \phi_{1}\right)-(1-\gamma) \nabla^{2} \phi_{0} \frac{\partial \phi_{1}}{\partial t}-\frac{1}{2}(1-\gamma) \\
& \times\left[\left(\nabla \phi_{0}\right)^{2}-M^{2}\right] \nabla^{2} \phi_{1}-(1-\gamma)\left(\nabla \phi_{0} \cdot \nabla \phi_{1}\right) \nabla^{2} \phi_{0} \\
& +\frac{1}{2}\left(\nabla \phi_{1} \cdot \nabla\right)\left(\nabla \phi_{0}\right)^{2}+\left(\nabla \phi_{0} \cdot \nabla\right)\left(\nabla \phi_{0} \cdot \nabla \phi_{1}\right),
\end{aligned}
$$

$\frac{\partial \phi_{1}}{\partial y}=\epsilon k_{w} \frac{\partial \phi_{1}}{\partial x} \cos \left(k_{w} x\right)$ at $y=\epsilon \sin \left(k_{w} x\right)$,

$\frac{\partial \phi_{1}}{\partial y}=\epsilon \alpha k_{w} \frac{\partial \phi_{1}}{\partial x} \cos \left(k_{w} x+\theta\right), \quad$ at $y=1+\epsilon \alpha \sin \left(k_{w} x+\theta\right)$.

Since the average mean flow is assumed to be uniform having a Mach number $M$, the mean flow can be expanded as 


$$
\phi_{0}=M x+\epsilon \phi_{01}(x, y)+\cdots .
$$

Substituting Eq. 13 into Eqs. 7-9, expanding for small $\epsilon$, and equating the coefficients of $\epsilon$ on both sides, we obtain

$$
\begin{aligned}
& \frac{\partial^{2} \phi_{01}}{\partial y^{2}}+\left(1-M^{2}\right) \frac{\partial^{2} \phi_{01}}{\partial x^{2}}=0, \\
& \frac{\partial \phi_{01}}{\partial y}=M k_{w} \cos \left(k_{w} x\right), \quad \text { at } y=0, \\
& \frac{\partial \phi_{01}}{\partial y}=\alpha M k_{w} \cos \left(k_{w} x+\theta\right), \quad \text { at } y=1 .
\end{aligned}
$$

The solution of Eqs. $14-16$ is

$$
\begin{aligned}
\phi_{01}= & \frac{M k_{w}}{\kappa_{w} \sinh \left(\kappa_{w}\right)}\left\{\left[\sinh \left(\kappa_{w}\right) \sinh \left(\kappa_{w} y\right)-\cosh \left(\kappa_{w}\right) \cosh \left(\kappa_{w} y\right)\right]\right. \\
& \left.\times \cos \left(k_{w} x\right)+\alpha \cosh \left(\kappa_{w} y\right) \cos \left(k_{w} x+\theta\right)\right\}
\end{aligned}
$$

where

$$
\kappa_{w}^{2}=k_{w}^{2}\left(1-M^{2}\right) .
$$

\section{A UNIFORM EXPANSION NEAR RESONANT FREQUENCIES}

Following the procedure of Ref. 5, one can show that straightforward expansions of the form

$$
\phi_{1}(x, y, t)=\phi_{10}(x, y, t)+\epsilon \phi_{11}(x, y, t)+\cdots
$$

for the solutions of Eqs. 10-12 break down for all frequencies such that

$$
k_{w} \approx k_{n} \pm k_{m},
$$

where $k_{n}$ and $k_{m}$ are the wavenumbers of any two propagating modes in the unperturbed duct (they are defined in Eq. 31). The resonant case $k_{w} \approx k_{n}+k_{m}$ occurs only for standing waves, while the other case occurs for both standing and traveling waves. In this paper, we consider traveling waves only.

To determine a uniform first-order expansion for the case $k_{w} \sim k_{n}-k_{m}$, we use the method of multiple scales and let

$$
\phi_{1}(x, y, t)=\phi_{10}\left(x_{0}, x_{1}, y, t\right)+\epsilon \phi_{11}\left(x_{0}, x_{1}, y, t\right)+\cdots,
$$

where $x_{0}=x$ is a length scale of the order of the wavelength and $x_{1}=\epsilon x$ is a long scale characterizing the amplitude and phase modulations. Moreover, we express the nearness of $k_{w}$ to $k_{n}-k_{m}$ by introducing the detuning parameter $\sigma$ according to

$$
k_{w}=k_{n}-k_{m}+\epsilon \sigma .
$$

Substituting Eqs. 13 and 21 into Eqs. 10-12 and equating coefficients of like powers of $\epsilon$ on both sides, we obtain:

Order $\epsilon^{0}$ :

$\mathcal{L}\left(\phi_{10}\right) \equiv \frac{\partial^{2} \phi_{10}}{\partial y^{2}}+\left(1-M^{2}\right) \frac{\partial^{2} \phi_{10}}{\partial x_{0}^{2}}-\frac{\partial^{2} \phi_{10}}{\partial t^{2}}-2 M \frac{\partial^{2} \phi_{10}}{\partial x_{0} \partial t}=0$

$\frac{\partial \phi_{10}}{\partial y}=0$, at $y=0$

$$
\frac{\partial \phi_{10}}{\partial y}=0, \text { at } y=1
$$

Order $\epsilon$ :

$$
\begin{aligned}
& \mathscr{L}\left(\phi_{11}\right)= 2 \frac{\partial}{\partial t}\left[\nabla_{0} \phi_{01} \cdot \nabla_{0} \phi_{10}\right]-(1-\gamma) \nabla_{0}^{2} \phi_{01}\left[M \frac{\partial \phi_{10}}{\partial x_{0}}+\frac{\partial \phi_{10}}{\partial t}\right] \\
&-(1-\gamma) M \frac{\partial \phi_{01}}{\partial x_{0}} \nabla_{0}^{2} \phi_{10}+2 M \frac{\partial}{\partial x_{0}}\left(\nabla_{0} \phi_{01} \cdot \nabla_{0} \phi_{10}\right) \\
&-2\left(1-M^{2}\right) \frac{\partial^{2} \phi_{10}}{\partial x_{0} \partial x_{1}}+2 M \frac{\partial^{2} \phi_{10}}{\partial x_{1} \partial t}, \\
& \frac{\partial \phi_{11}}{\partial y}=- \frac{\partial^{2} \phi_{10}}{\partial y^{2}} \sin \left(k_{w} x_{0}\right)+k_{w} \frac{\partial \phi_{10}}{\partial x_{0}} \cos \left(k_{w} x_{0}\right), \text { at } y=0, \\
& \frac{\partial \phi_{11}}{\partial y}=-\alpha \frac{\partial^{2} \phi_{10}}{\partial y^{2}} \sin \left(k_{w} x_{0}+\theta\right)+\alpha k_{w} \frac{\partial \phi_{10}}{\partial x_{0}} \cos \left(k_{w} x_{0}+\theta\right), \\
& \text { at } y=1,
\end{aligned}
$$

where

$$
\nabla_{0}=\frac{\partial}{\partial x_{0}} \overrightarrow{\mathbf{e}}_{x}+\frac{\partial}{\partial y} \overrightarrow{\mathrm{e}}_{y} .
$$

Following the procedure of Ref. 5, the solution of Eqs. 23-25 is taken to consist of the two interacting modes; that is,

$$
\begin{aligned}
\phi_{10}= & A_{m}\left(x_{1}\right) \cos (m \pi y) \exp \left[i\left(k_{m} x_{0}-\omega t\right)\right]+A_{n}\left(x_{1}\right) \cos (n \pi y) \\
& \times \exp \left[i\left(k_{n} x_{0}-\omega t\right)\right],
\end{aligned}
$$

where $\omega$ is the frequency of the sound and

$$
\left(\omega-M k_{j}\right)^{2}-k_{j}^{2}=j^{2} \pi^{2} .
$$

The functions $A_{m}$ and $A_{n}$ are arbitrary at this level of approximation; they are determined below from the solvability conditions of the first-order problem consisting of Eqs. 26-28.

Substituting for $\phi_{01}$ and $\phi_{10}$ from Eqs. 17 and 30 into Eqs. 26-28 yields

$$
\begin{aligned}
\mathscr{L}\left(\phi_{11}\right)= & \sum_{j=m, n}-2 i\left[\left(1-M^{2}\right) k_{j}+M \omega\right] \frac{d A_{j}}{d x_{1}} \cos (j \pi y) \\
& \times \exp \left[i\left(k_{j} x_{0}-\omega t\right)\right]+i \sum_{j=m, n} A_{j}\left\{F _ { 1 j } ( y ) \operatorname { e x p } \left[i\left(k_{j}+k_{w}\right)\right.\right. \\
& \left.\left.\times x_{0}-i \omega t\right]+F_{2 j}(y) \exp \left[i\left(k_{j}-k_{w}\right) x_{0}-i \omega t\right]\right\}, \\
\frac{\partial \phi_{11}}{\partial y}= & \frac{1}{2} i \sum_{j=m, n} A_{j}\left(k_{j} k_{w}-j^{2} \pi^{2}\right) \exp \left[i\left(k_{j}+k_{w}\right) x_{0}\right] \\
+ & \frac{1}{2} i \sum_{j=m, n} A_{j}\left(k_{j} k_{w}+j^{2} \pi^{2}\right) \exp \left[i\left(k_{j}-k_{w}\right) x_{0}\right], \text { at } y=0, \\
\frac{\partial \phi_{11}}{\partial y}= & \frac{1}{2} i \alpha \sum_{j=m, n} A_{j}\left(k_{j} k_{w}-j^{2} \pi^{2}\right) \cos (j \pi) \exp \left[i\left(k_{j}+k_{w}\right) x_{0}+i \theta\right] \\
& +\frac{1}{2} i \sum_{j=m, n} A_{j}\left(k_{j} k_{w}+j^{2} \pi^{2}\right) \cos (j \pi) \exp \left[i\left(k_{j}-k_{w}\right) x_{0}-i \theta\right], \\
& \text { at } y=1,
\end{aligned}
$$

where the $F$ 's are defined in Appendix A.

The straightforward first-order expansion can be obtained by solving Eqs. 32-34 with $d A_{j} / d x_{1}=0$. However, the resulting particular solution contains small divisor terms $\mathrm{s}^{5}$ whenever $k_{w} \approx k_{n}-k_{m}$. These small divisor terms 
make the supposedly small correction term $\epsilon \phi_{11}$ much larger than the preceding term $\phi_{10}$ in Eq. 21 and thereby invalidate the straightforward expansion. To obtain a uniform expansion, we chose the $A_{j}$ to remove any small divisor terms. To accomplish this, we use Eq. 22 to express $\left(k_{w}+k_{m}\right) x_{0}$ and $\left(k_{n}-k_{w}\right) x_{0}$ as

$$
\begin{aligned}
& \left(k_{w}+k_{m}\right) x_{0}=k_{n} x_{0}+\sigma x_{1}, \\
& \left(k_{n}-k_{w}\right) x_{0}=k_{m} x_{0}-\sigma x_{1} .
\end{aligned}
$$

Since the homogeneous part of the first-order problem (Eqs. 32-34) is the same as the zeroth-order problem (Eqs. 23-25), the first-order problem has a solution if, and only if, solvability conditions are satisfied. To determine these solvability conditions, we seek a particular solution of the form

$$
\phi_{11}=i \sum_{j} \psi_{j}\left(y ; x_{1}\right) \exp \left[i\left(k_{j} x_{0}-\omega t\right)\right] .
$$

Substituting Eq. 36 into Eqs. 32-34, using Eqs. 35, and equating the coefficients of $\exp \left[i\left(k_{j} x_{0}-\omega t\right)\right]$ on both sides, we obtain

$$
\begin{aligned}
& \partial^{2} \psi_{m} / \partial y^{2}+m^{2} \pi^{2} \psi_{m}=-2\left[\left(1-M^{2}\right) k_{m}+M \omega\right] A_{m}^{\prime} \cos (m \pi y) \\
& +A_{n} F_{2 n}(y) \exp \left(-i \sigma x_{1}\right) \text {, } \\
& \partial \psi_{m} / \partial y=\frac{1}{2} A_{n}\left(k_{n} k_{w}+n^{2} \pi^{2}\right) \exp \left(-i \sigma x_{1}\right), \quad \text { at } y=0 \text {, } \\
& \partial \psi_{m} / \partial y=\frac{1}{2} \alpha A_{n}\left(k_{n} k_{w}+n^{2} \pi^{2}\right) \cos (n \pi) \exp \left[-i\left(\sigma x_{1}+\theta\right)\right] \text {, } \\
& \text { at } y=1 \text {, } \\
& \partial^{2} \psi_{n} / \partial y^{2}+n^{2} \pi^{2} \psi_{n}=-2\left[\left(1-M^{2}\right) k_{n}+M \omega\right] A_{n}^{\prime} \cos (n \pi y) \\
& +A_{m} F_{1 m}(y) \exp \left(i \sigma x_{1}\right) \text {, } \\
& \partial \psi_{n} / \partial y=\frac{1}{2} A_{m}\left(k_{m} k_{w}-m^{2} \pi^{2}\right) \exp \left(i \sigma x_{1}\right), \quad \text { at } y=0 \text {, } \\
& \partial \psi_{n} / \partial y=\frac{1}{2} \alpha A_{m}\left(k_{m} k_{w}-m^{2} \pi^{2}\right) \cos (m \pi) \exp \left[i\left(\sigma x_{1}+\theta\right)\right] \\
& \text { at } y=1 \text {, }
\end{aligned}
$$

where primes denote differentiation with respect to $x_{1}$. Thus, determining the solvability conditions for Eqs. 32-34 is transformed into that of determining the solvability conditions for Eqs. 37-42.

To determine the solvability conditions for Eqs. 3739 , we multiply Eq. 37 by $\cos (m \pi y)$, integrate the result by parts, and obtain

$$
\begin{aligned}
\left.\frac{\partial \psi_{m}}{\partial y} \cos (m \pi y)\right|_{0} ^{1}= & -\left[\left(1-M^{2}\right) k_{m}+M \omega\right] A_{m}^{\prime}+A_{n} \exp \left(-i \sigma x_{1}\right) \\
& \times \int_{0}^{1} F_{2 n}(y) \cos (m \pi y) d y .
\end{aligned}
$$

Using the boundary conditions 38 and 39, we rewrite Eq. 43 as

$$
A_{m}^{\prime}=\Lambda_{1} A_{n} \exp \left(-i \sigma x_{1}\right)
$$

where

$$
\begin{aligned}
\Lambda_{1}=\left[\left(1-M^{2}\right) k_{m}+M \omega\right]^{-1} & \left\{\frac{1}{2}\left(k_{n} k_{m}+n^{2} \pi^{2}\right)\left[1-\alpha(-1)^{n+m} e^{-i \theta}\right]\right. \\
& \left.+\int_{0}^{1} F_{2 n}(y) \cos (m \pi y) d y\right\} .
\end{aligned}
$$

Similarly, the solvability condition for Eqs. $40-42$ is

$$
A_{n}^{\prime}=\Lambda_{2} A_{m} \exp \left(i \sigma x_{1}\right),
$$

$$
\begin{aligned}
& \text { where } \\
& \begin{aligned}
\Lambda_{2}=\left[\left(1-M^{2}\right) k_{n}+M \omega\right]^{-1} & \left\{\frac{1}{2}\left(k_{m} k_{w}-m^{2} \pi^{2}\right)\left[1-\alpha(-1)^{m+n} e^{i \theta}\right]\right. \\
& \left.+\int_{0}^{1} F_{1 m}(y) \cos (n \pi y) d y\right\} .
\end{aligned}
\end{aligned}
$$

Equations 44 and 46 admit solutions of the form

$$
A_{m}=a_{m} \exp \left(i s x_{1}\right), A_{n}=a_{n} \exp \left[i(s+\sigma) x_{1}\right],
$$

with $a_{m}$ and $a_{n}$ being constants provided that

$$
s^{2}+\sigma s+\Lambda_{1} \Lambda_{2}=0 \text {. }
$$

The solution of Eq. 49 is

$$
s=\frac{1}{2}\left[-\sigma \pm\left(\sigma^{2}-4 \Lambda_{1} \Lambda_{2}\right)^{1 / 2}\right] .
$$

Substituting for $F_{1 m}$ and $F_{2 n}$ from Appendix A into Eqs. 45 and 47 , carrying out the integrations, and letting $k_{w} \approx k_{n}-k_{m}$, we can express $4 \Lambda_{1} \Lambda_{2}$ as

$$
\begin{aligned}
4 \Lambda_{1} \Lambda_{2}= & {\left[1+\alpha^{2}-2 \alpha(-1)^{m+n} \cos \theta\right]\left[\left(1-M^{2}\right) k_{n}+M \omega\right]^{-1} } \\
& \times\left[\left(1-M^{2}\right) k_{m}+M \omega\right]^{-1} \Gamma_{1} \Gamma_{2},
\end{aligned}
$$

where the $\Gamma$ 's are real and defined in Appendix A. Since $\Lambda_{1} \Lambda_{2}$ is real, Eq. 50 shows that $s$ is real and the waves travel without being attenuated or being unstable when $\sigma^{2}-4 \Lambda_{1} \Lambda_{2}$ is positive; otherwise $s$ is complex. In the latter case, decaying as well as growing wave solutions are possible, depending on the initial conditions. In both cases, the $m$ th mode cannot exist in the duct without strongly exciting the $n$th mode. As $M \rightarrow 0$,

$$
\begin{aligned}
4 \Lambda_{1} \Lambda_{2} \rightarrow & {\left[1+\alpha^{2}-2 \alpha(-1)^{m+n} \cos \theta\right]\left(k_{m} k_{n}\right)^{-1}\left(k_{n} k_{w}+n^{2} \pi^{2}\right) } \\
& \times\left(k_{m} k_{w}-m^{2} \pi^{2}\right) .
\end{aligned}
$$

Hence, the sign of $\Lambda_{1} \Lambda_{2}$ depends on the sign of the last factor in Eq. 52. Using Eqs. 22 and 31 with $M=0$, one can show that

$$
k_{m} k_{n}-m^{2} \pi^{2}=-\left(k_{n} k_{w}+n^{2} \pi^{2}\right)+\epsilon \sigma\left(k_{n}+k_{m}\right) .
$$

Since the second term on the right-hand side of Eq. 53 is small compared with the first term, $k_{m} k_{w}-m^{2} \pi^{2}<0$. Hence, $\Lambda_{1} \Lambda_{2}<0$. Consequently, the two modes are strongly coupled and travel unattenuated along the duct in agreement with the result of Ref. 5.

Carrying out the expansion to higher order, one expects to encounter resonances whenever $k_{m}-k_{n} \approx r k_{w}$, where $r$ is an integer. Hence, the $m$ th mode cannot exist in the duct without strongly exciting the $n$th mode and vice versa.

\section{ACKNOWLEDGMENT}

The comments of Dr. John E. Kaiser are greatly appreciated.

\section{APPENDIX A}

$$
\begin{aligned}
F_{1 j}(y)= & \left(M k_{j}+M k_{w}-\omega\right)\left\{\left[-k_{w} k_{f}+\frac{1}{2}(1-\gamma) M k_{w}\left(M k_{j}-\omega\right)\right]\right. \\
& \times \cos (j \pi y)\left[c_{1} \sinh \left(\kappa_{w} y\right)-c_{2} \cosh \left(\kappa_{w} y\right)\right]-j \pi \kappa_{w} \\
& \left.\times \sin (j \pi y)\left[c_{1} \cosh \left(\kappa_{w} y\right)-c_{2} \sinh \left(\kappa_{w} y\right)\right]\right\}, \\
F_{2 j}= & \left(M k_{j}-M k_{w}-\omega\right)\left\{\left[k_{w} k_{j}-\frac{1}{2}(1-\gamma) M k_{w}\left(M k_{j}-\omega\right)\right]\right. \\
& \times \cos (j \pi y)\left[c_{1} \sinh \left(\kappa_{w} y\right)-\bar{c}_{2} \cosh \left(\kappa_{w} y\right)\right]-j \pi \kappa_{w} \sin (j \pi y)
\end{aligned}
$$


$\left.\times\left[c_{1} \cosh \left(\kappa_{w} y\right)-\bar{c}_{2} \sinh \left(\kappa_{w} y\right)\right]\right\}$,

(A2)

where

$\left(c_{1}, c_{2}\right)=\frac{M k_{w}}{\kappa_{w} \sinh \left(\kappa_{w}\right)}\left[\sinh \left(\kappa_{w}\right), \cosh \left(\kappa_{w}\right)-\alpha \exp (i \theta)\right]$.

$\Gamma_{1}=k_{n} k_{w}+n^{2} \pi^{2}+M k_{w}\left(\omega-M k_{m}\right)\left\{\left[k_{n} k_{w}+\frac{1}{2}(1-\gamma) M k_{w}\right.\right.$ $\left.\left.\times\left(\omega-M k_{n}\right)\right] \xi_{1}+n \pi^{2} \xi_{3}\right\}$,

$\Gamma_{2}=k_{m} k_{w}-m^{2} \pi^{2}-M k_{w}\left(\omega-M k_{n}\right)\left\{\left[k_{m} k_{w}+\frac{1}{2}(1-\gamma) M k_{w}\right.\right.$

$$
\left.\left.\times\left(\omega-M k_{n}\right)\right] \xi_{1}+m \pi^{2} \xi_{2}\right\},
$$

where

$$
\xi_{1}=\eta_{1}+\eta_{2}
$$

$$
\begin{aligned}
& \xi_{2}=(m+n) \eta_{1}+(m-n) \eta_{2}, \\
& \xi_{3}=(m+n) \eta_{1}-(m-n) \eta_{2}, \\
& \eta_{1}=\left[\kappa_{w}^{2}+\pi^{2}(m+n)^{2}\right]^{-1}, \\
& \eta_{2}=\left[\kappa_{w}^{2}+\pi_{2}^{2}(m-n)^{2}\right]^{-1} .
\end{aligned}
$$

${ }^{1} \mathrm{H}$. S. Tsien, in Fundamentals of Gasdynamics, H. W. Emmons, Ed. (Princeton U. P., Princeton, NJ, 1958), pp. 36-37.

${ }^{2}$ M. A. Isakovitch, Akust. Zhur. 3, 37-45 (1957).

${ }^{3}$ J. S. Samuels, J. Acoust. Soc. Am. 31, 319-325 (1959).

${ }^{4}$ R. F. Salant, J. Acoust. Soc. Am. 53, 504-507 (1973).

${ }^{5}$ A. H. Nayfeh, J. Acoust. Soc. Am. 56, 768-770 (1974).

${ }^{6}$ A. H. Nayfeh, Perturbation Methods (Wiley-Interscience, New York, 1973), Chap. 6. 